correlation $=0.87, \mathrm{p}<0.001)$ extracted two discriminant functions that accounted for $95.4 \%$ of the variance, with the first function, reflecting global cognitive function, accounting for $83 \%$. Reclassification using the canonical variables was $72.9 \%$ correct. Predicted values from the first discriminant function revealed that the interaction between health status and physical fitness was due to the latter improving global cognitive function in the dementia group $[\mathrm{t}(25)=2.90, \mathrm{p}<0.01]$, but not in the healthy group $(\mathrm{p}>0.10)$. A regression analysis howed that although global functioning decreased with the number of years since dementia diagnosis [unstandardised regression coefficients $(b)=-0.25,95 \%$ CI $(-0.47,-0.04), p<0.05]$, physical fitness was still associated with improved cognitive functioning $[b=1.54,95 \%$ CI $(.49,2.59)]$, with no interaction $(p>0.05)$.

Conclusion Physical fitness was associated with improved cognitive functioning, particularly for individuals with dementia. This benefit was present independent of exercise history, age, or duration of dementia. These findings provide empirical support for the development of fitness programmes for dementia patients to offset the cognitive deterioration associated with the condition.

\section{P58 BARRIERS AND FACILITATORS TO IMPLEMENTATION OF DIET AND PHYSICAL ACTIVITY INTERVENTIONS IN SCHOOLS-A DEDIPAC (DETERMINANTS OF DIET AND PHYSICAL ACTIVITY) QUALITATIVE STUDY}

${ }^{1} \mathrm{CB}$ Hayes ${ }^{*},{ }^{1} \mathrm{MP}$ O'Shea, ${ }^{2} \mathrm{~K}$ Horodyska, ${ }^{2} \mathrm{~A}$ Luszczynska, ${ }^{3} \mathrm{UJ}$ Langøien, ${ }^{4} \mathrm{G}$ Roos, ${ }^{5} \mathrm{~S}$ Muellman, ${ }^{5} \mathrm{CR}$ Pischke, ${ }^{6} \mathrm{I}$ De Bourdeaudhui, ${ }^{7} \mathrm{~J}$ Brug. ${ }^{1}$ Public Health and Primary Care, Trinity College Dublin, Dublin, Ireland; ' ${ }^{2}$ sychology, SWPS University of Social Sciences and Humanities, Wroclaw, Poland; ${ }^{3}$ Physical Education, Norwegian School of Sports Science, Oslo, Norway; ${ }^{4}$ Consumption Research Norway, SIFO, Oslo and Akershus University College of Applied Sciences, Oslo, Norway; ${ }^{5}$ Leibniz Institute for Prevention Research and Epidemiology, BIPS, Bremen, Germany; ${ }^{6}$ Movement and Sports Sciences, Ghent University, Ghent, Belgium; ' Social and Behavioural Sciences, VU University Medical Centre (VUmc), Amsterdam, Netherlands

\subsection{6/jech-2017-SSMAbstracts. 159}

Background This case study was undertaken in Ireland as part of the European DEterminants of DIet and Physical Activity (DEDIPAC) Knowledge Hub. Two national interventions were chosen based on predetermined selection criteria: a Healthy Eating Programme (HEP) to encourage primary schoolchildren to consume more fruit and vegetables, and a Travel to School Programme, (TSP) to promote sustainable modes of transport, car-pooling and public transport use in primary and secondary schools. The HEP is EU and government funded, the TSP entirely government funded. TSP adopts a flexible approach where schools can set their travel targets. School coordinators (teachers) cascade both programmes to classroom teachers.

Methods Seven of eight schools invited to participate based on predetermined criteria took part in the study. Face-to-face interviews $(n=15)$ were conducted with teachers, project managers and key stakeholders using a topic guide developed by the international DEDIPAC team and informed by a prior systematic umbrella review of conditions influencing implementation. Data were coded in NVIVO using a common categorization matrix and thematic analysis carried out using parameters of the RE-AIM (Reach, Effectiveness, Adoption, Implementation, Maintenance) evaluation framework.

Results Good working relationships were critical to adoption, successful implementation and sustainability in line with findings from case studies in other EU countries. Organisational and leadership ability of coordinators was key to successful delivery. Incentives and rewards acted as motivators to engage children's interest, which motivated teacher and parent involvement. Particular challenges faced by the TSP included a lack of funding security and timetable constraints within secondary schools. HEP was based on well-funded external research with clearly defined core components and has been frequently externally evaluated. TSP core components were broad rather than specific, implementation was flexible and there was a lack of agreement among stakeholders on how targets were set and the accuracy of these.

Conclusion Good relationships, organisational and leadership ability, and secure funding were key conditions for implementation, sustainability and dissemination of promising public health interventions. The findings have informed the DEDIPAC-KH Pan European Toolbox set up for researchers and practitioners who want to develop, evaluate or implement multicomponent interventions on physical activity, sedentary behaviour or dietary behaviour.

\section{P59 LIFELAB SOUTHAMPTON: IMPROVING SCIENCE LITERACY AS A TOOL FOR INCREASING HEALTH LITERACY IN TEENAGERS - A PILOT CLUSTER- RANDOMISED CONTROLLED TRIAL}

\begin{abstract}
${ }^{1,2} \mathrm{KS}$ Woods-Townsend, ${ }^{3} \mathrm{H}$ Aiston, ${ }^{1} \mathrm{~L}$ Bagust, ${ }^{1} \mathrm{H}$ Davey, ${ }^{1} \mathrm{D}$ Lovelock, ${ }^{1} \mathrm{~A}$ Christodoulou, 1,4JB Griffiths, ${ }^{1} \mathrm{MM}$ Grace, $2,5,6 \mathrm{KM}$ Godfrey, ${ }^{2,6} \mathrm{MA}$ Hanson, 2,5 HM Inskip*. 'Southampton Education School, University of Southampton, Southampton, UK; ${ }^{2}$ NIHR Southampton Biomedical Research Centre, University of Southampton and University Hospital Southampton NHS Foundati, Southampton, UK; ${ }^{3}$ Science, St Anne's Catholic School, Southampton, UK; ${ }^{4}$ Mathematics and Science Learning Centre, University of Southampton, Southampton, UK; ${ }^{5} M R C$ Lifecourse Epidemiology Unit, University of Southampton, Southampton, UK; ${ }^{6}$ Human Development and Health Academic Unit, University of Southampton, Southampton, UK
\end{abstract}

10.1136/jech-2017-SSMAbstracts. 160

Background Behavioural risk factors are the largest contributor to the non-communicable disease burden, and those of parents can affect prenatal and infant development with lasting impact on children's long-term health. Adolescence offers a window of opportunity during which improvements in health behaviours would not only benefit long-term health of individuals, but also enable them to be better prepared for parenthood and pass better health prospects to their children. We have developed an educational intervention, LifeLab, based around a purpose-built laboratory in University Hospital Southampton with support from teachers, to engage adolescents in understanding effects of their health behaviours for themselves and their future children.

Aims To assess whether engaging adolescents with the science behind health messages, thus improving their science literacy, increases their health literacy and hence their health behaviours.

Methods In a pilot study, in preparation for a large cluster randomised trial of LifeLab, we recruited six schools. Three were randomised to the LifeLab intervention and three to control, with 392 students completing online questionnaires at baseline and 12 months follow up. Summary statistics were used to examine differences between groups. The categorical outcome variables were dichotomised and Poisson regression with robust variance used to obtain prevalence rate ratios (PRRs) for the outcome in relation to the intervention, 